\title{
Minimization of Ga Induced FIB Damage Using Low Energy Clean-up
}

\author{
K. Thompson*, B. Gorman ${ }^{* *}$, D. J. Larson ${ }^{*}$, Brandon van Leer ${ }^{* * *}$ and Liang Hong ${ }^{* * *}$ \\ * Imago Scientific Instruments Corp. 6300 Enterprise Ln, Suite 100, Madison, WI 53719 \\ ** University of North Texas, Denton TX \\ *** FEI company, Hillsboro OR
}

The use of a focused ion beam (FIB) is essential in the fabrication of high quality atom-probe specimens [1-2]. The primary drawback to FIB sharpening is the damage inflicted at the apex of the atom probe specimen by the high energy $\mathrm{Ga}^{+}$ions. This paper presents a method to minimize this ion damage using a low energy FIB process in the final stage of the specimen preparation.

Figure 1 displays the theoretical range, obtained via SRIM calculations [3], of $\mathrm{Ga}^{+}$in both $\mathrm{Si}$ and $\mathrm{Pt}$ for various accelerating energies normal to the surface of the sample. The implantation range into Si is approximately four times that for $\mathrm{Pt}$ at $30 \mathrm{keV} \mathrm{Ga}$, which is used by most FIB systems. The damage at such energies is sufficient to amorphize the top several tens of nanometers of the specimen. While this can be avoided by applying a thick $(\sim 50 \mathrm{~nm})$ metal cap on the specimen before FIB sharpening, one must then atom-probe through the damaged cap region before reaching the area of interest. This extends the analysis time and increases the probability of sample failure. A post-FIB clean-up step may be employed to minimize this amorphous region. While this clean-up could be performed in a lower energy, broad ion beam system [4], this inhibits the easy positioning of the location of the region of interest. A more effective method is to perform the low energy $(2-$ $10 \mathrm{keV}$ ) clean-up step in the FIB immediately after the specimen sharpening process is complete [5].

The depth of Ga penetration as a function of the accelerating energy of the low keV clean-up process was investigated. First, Si-based atom-probe specimens were FIB sharpened at $30 \mathrm{keV}$. Specimens were then cleaned up with either the $2 \mathrm{keV}$ or the $5 \mathrm{keV}$ beam. Finally, the tips were analyzed in the LEAP3000X system in order to quantify the extent of Ga penetration. No protective metal FIB caps were applied to any of the specimens. The results, Figs. $2-4$, demonstrate that the low keV clean-up process dramatically reduces both the quantity and the depth of penetration of the Ga in the specimen. Fig. 2 displays the 1-D composition profile for $\mathrm{Ga}$ into $\mathrm{Si}$. The profile was obtained by analyzing a $10 \mathrm{~nm}$ cylinder of data aligned along the analysis direction. The analysis cylinder was sliced into $0.5 \mathrm{~nm}$ thick volumes, and the $\mathrm{Ga}$ atoms and $\mathrm{Si}$ atoms in each slice were counted. The 30 $\mathrm{keV}$ standard beam energy has a peak Ga concentration of 12 at.\% and a maximum penetration depth of $\sim 40 \mathrm{~nm}$. By comparison, the $5 \mathrm{keV}$ clean-up beam has a peak Ga concentration of 2 at. $\%$ and a penetration depth of $\sim 5 \mathrm{~nm}$. The $2 \mathrm{keV}$ clean-up beam had so little Ga that it was impossible to quantify the $\mathrm{Ga}$ content in this manner. In order to more accurately quantify the $\mathrm{Ga}$ penetration from the $2 \mathrm{keV}$ beam, the mass spectrum for the first 1 million Si atoms collected is shown in Figs. 3 and 4. Figure 3 shows the mass spectrum for the 30, 5 and $2 \mathrm{keV}$ beams. For clarity, Fig. 4 shows the same mass spectrum for the 5 and $2 \mathrm{keV}$ beams only. The $30 \mathrm{keV}$ beam deposits 16,603 Ga atoms in the first 1 million Si atoms. By comparison the 5 and $2 \mathrm{keV}$ beam deposited 893 and $16 \mathrm{Ga}$ atoms, respectively. It is important to note that an atom probe specimen evaporates $2-3 \mathrm{~nm}$ of material in an uncontrolled fashion as the tip shape forms. During this time, meaningful data is not collected. As a result, the Ga concentration in the first few $\mathrm{nm}$ of the surface is not representative of the actual surface but rather that a few nm into the sample. Applying a cap layer to the surface of the tip before beginning the evaporation would avoid this Ga dose loss. 
It is evident that the low $\mathrm{keV}$ clean-up process virtually eliminates the Ga damaged regions of $\mathrm{Si}$ based atom probe specimens. The result is a high quality, damage free atom probe specimen whose region of interest is reached shortly after the beginning of the atom probe analysis.

[1] D. J. Larson et al., Acta Mater. 52(10) (2004) 2847.

[2] M. K. Miller et al., Ultramicroscopy 102 (2005) 287.

[3] SRIM code available from J. F. Ziegler, IBM (www.srim.org).

[4] D. J. Larson et al., J.Vac. Sci. Tech. B 18(1) (2000) 328.

[5] L. A. Giannuzzi, R. Geurts, J. Ringnalda, Microscopy \& Microanalysis 1192 (2005) 828.

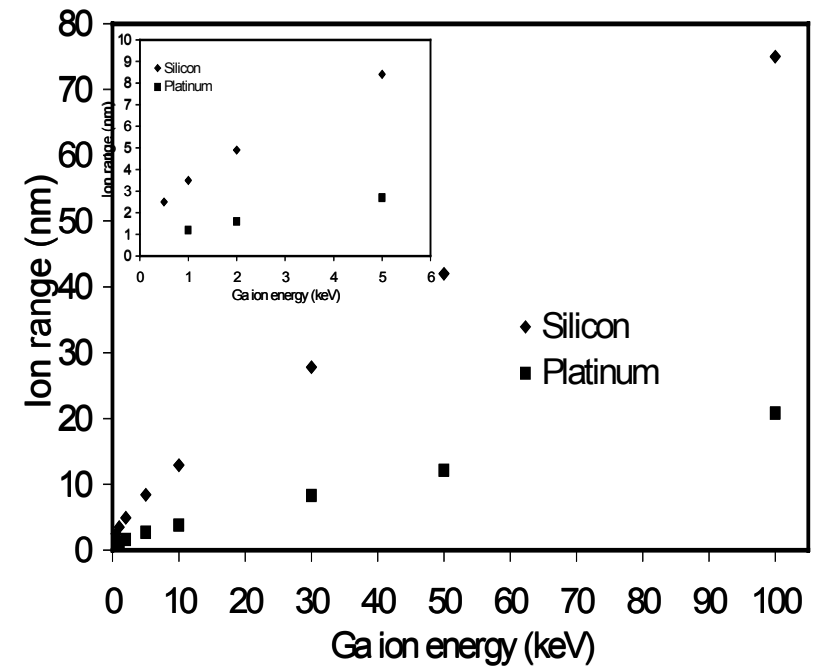

Fig. 1. Projected range for $\mathrm{Ga}^{+}$penetration in $\mathrm{Si}$ and $\mathrm{Pt}$; at normal incidence from SRIM calculations.

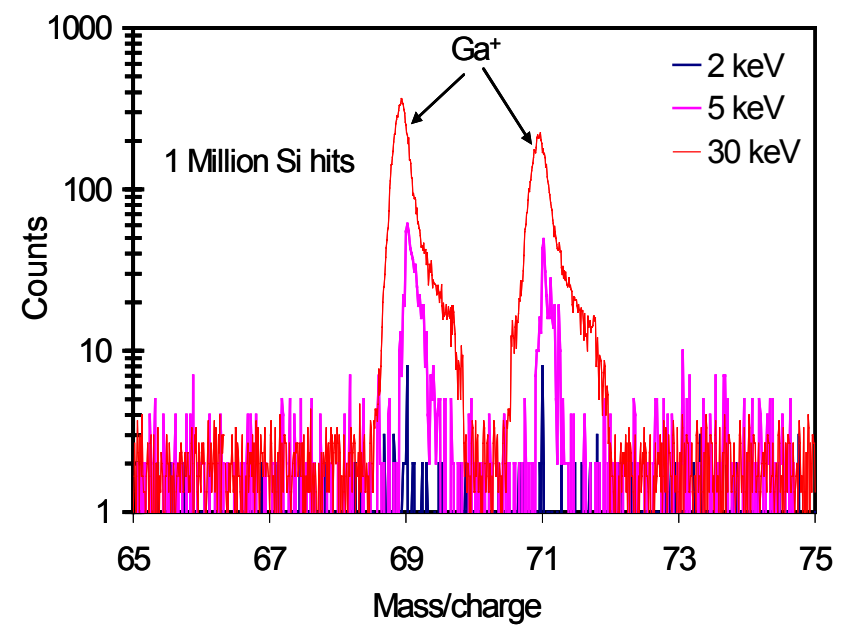

Fig. 3. Mass spectrum in the Ga region for the $2 \mathrm{keV}, 5$ $\mathrm{keV}$ and $30 \mathrm{keV}$ accelerating energies. 1 million $\mathrm{Si}$ atoms were sampled for each spectrum.

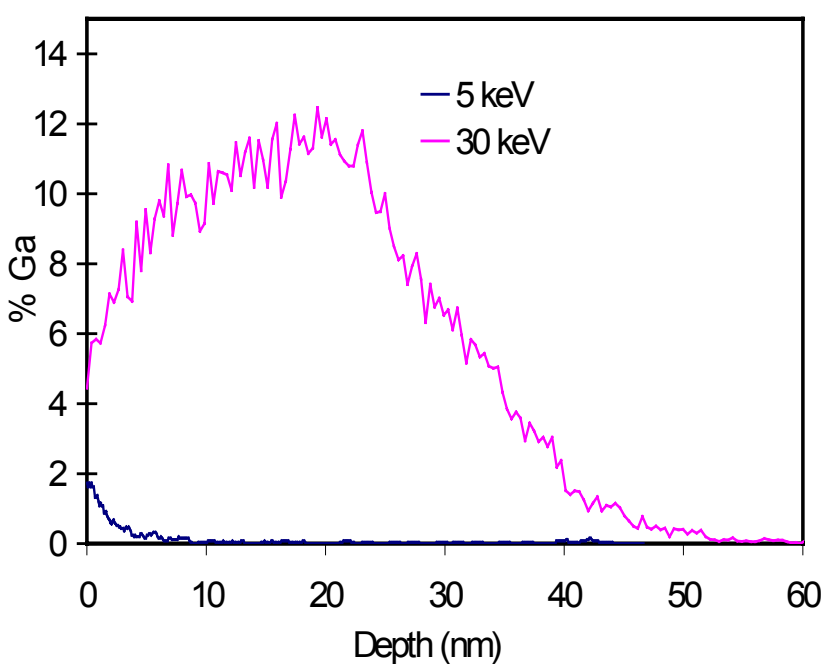

Fig. 2. $\mathrm{Ga}^{+}$implantation profile in $\mathrm{Si}$ for $30 \mathrm{keV}$ and 5 $\mathrm{keV}$ accelerating energies.

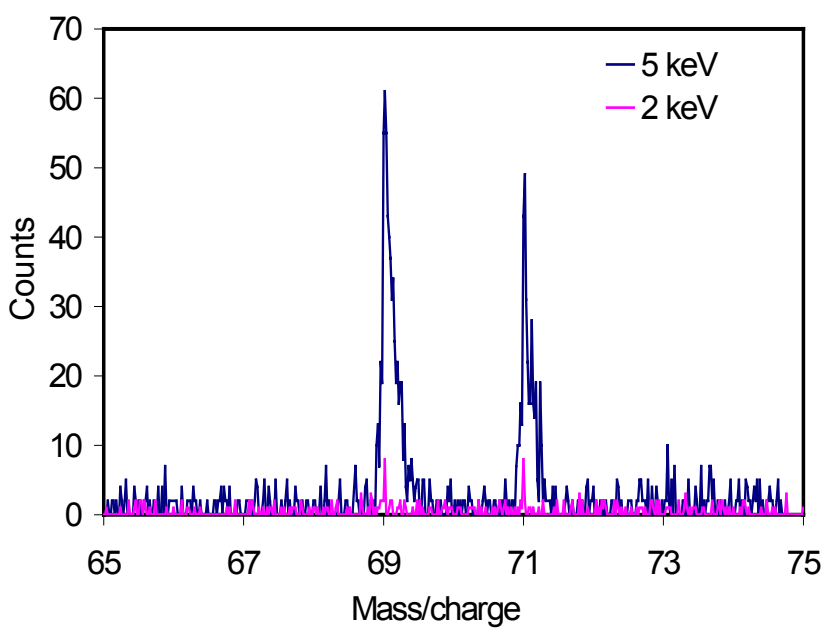

Fig. 4. Mass spectrum in the $\mathrm{Ga}$ region for the $2 \mathrm{keV}$ and $5 \mathrm{keV}$ accelerating energies. 1 million $\mathrm{Si}$ atoms were sampled for each spectrum. 